\title{
Evaluation of Some Sums of Polynomials in $\mathbb{F}_{q}[t]$
}

\author{
Adama Diene \\ Department of Mathematical Sciences, United Arab Emirates University, PO Box 17551, Al-Ain, UAE \\ Correspondence should be addressed to Adama Diene; adiene@uaeu.ac.ae
}

Received 5 September 2018; Accepted 4 November 2018; Published 9 July 2019

Academic Editor: Nazim I. Mahmudov

Copyright (c) 2019 Adama Diene. This is an open access article distributed under the Creative Commons Attribution License, which permits unrestricted use, distribution, and reproduction in any medium, provided the original work is properly cited.

We prove the polynomial analogues of some Liouville identities from elementary number theory. Consequently several sums defined over the finite fields $\mathbb{F}_{q}[t]$ are evaluated by combining the results obtained and some of the results from sums of reciprocals of polynomials over $\mathbb{F}_{q}[t]$.

\section{Introduction and Background}

Let $p$ be a prime number, $q=p^{e}$ for some positive integer $e$ and $\mathbb{F}_{q}$ the finite field with $q$ elements. It is well known that $\mathbb{F}_{q}[t]$ and the set of all integers $\mathbb{Z}$ share many similarities. Analogue of many results on $\mathbb{Z}$ has been proven for $\mathbb{F}_{q}[t]$. Both rings are principal ideal domains, both have the property that the residue class ring of any nonzero ideal is finite, both rings have infinitely many prime elements, and both rings have finitely many units. For the ring $\mathbb{Z}$, the units are \pm 1 and every nonzero integer is a multiple by a unit of a positive integer. Similarly, the units of $\mathbb{F}_{q}[t]$ are the nonzero scalars $\mathbb{F}_{q}^{*}$, and every nonzero polynomial in $\mathbb{F}_{q}[t]$ is a multiple by a unit of a monic polynomial. Thus, one may think that many other results, which hold for $\mathbb{Z}$, have their analogues in the ring $\mathbb{F}_{q}[t]$. This is indeed the case. For example, analogues of the little theorem of Fermat and Euler, Wilsons theorem, quadratic (and higher) reciprocity, the prime number theorem, and Dirichlet's theorem on primes in arithmetic progression and many more well-known theorems from elementary number theory have been proven true for $\mathbb{F}_{q}[t]$, see $[1]$.

In the nineteenth century, Joseph Liouville introduced a powerful new method into elementary number theory that allowed him to get many interesting identities. His approach was also used to solve well-known problems such as sums of squares, sums of triangular numbers, recurrence relations for divisor functions, convolution sums, and many others. Most of these problems are still today a subject of mathematical research.
In this present paper, we propose to study the polynomial analogue of some identities of Liouville in Williams [2, chapter 3]. More precisely, if $d(n)$ and $\sigma(n)$ represent respectively the number of divisors and the sum of divisors of $n$, and

$$
d_{k, m}(g):=\sum_{\substack{d \in \mathbb{N} \\ d \mid g \\ d \equiv k(\bmod m)}} 1, \quad n \in \mathbb{N},
$$

Liouville proved the following.

Theorem 1 (Liouville). Let $a \in \mathbb{Z}^{*}, b \in \mathbb{Z}, m \in \mathbb{N}$, and $n \in \mathbb{N}$. Set

$$
\begin{aligned}
g & :=\operatorname{gcd}(a, m), \\
a_{1} & :=\frac{a}{g}, \\
m_{1} & :=\frac{m}{g} .
\end{aligned}
$$

If $a_{2} \in 1,2, \ldots, m_{1}-1$ satisfies

$$
a_{1} a_{2} \equiv 1\left(\bmod m_{1}\right) \text {, }
$$

then

$$
\begin{aligned}
\sum_{\substack{d \in \mathbb{N} \\
d \mid n}}\left[\frac{a d+b}{m}\right]= & \frac{a}{m} \sigma(n)+\frac{b}{m} d(n) \\
& -\frac{1}{m} \sum_{\substack{l=1 \\
l=b(\bmod m)}}^{m-1} l d_{a_{2}(l-b) / g, m_{1}}(n),
\end{aligned}
$$

where $[x]$ represents the integer $n$ satisfying $n \leq x<n+1$. 
He also proved many other results related to Theorem 1 .

The main goal of this paper is to evaluate some sums of polynomials over $\mathbb{F}_{q}$ involving the polynomial analogue of Theorem 1. We also evaluate the average of some functions defined on the set of all monic polynomials over $\mathbb{F}_{q}$.

Throughout this paper, we let $A=\mathbb{F}_{q}[t]$. We define $M$, $M_{n}$, and $M_{<n}$ to be the subset of all monic polynomials in $A$, the subset of all monic polynomials in $A$ of degree $n$, and the subset of all monic polynomials in $A$ of degree less than $n$, respectively. We also let $P_{n}$ and $P_{<n}$ to be respectively the subset of all monic irreducible polynomials in $A$ of degree $n$ and the subset of all monic irreducible polynomials in $A$ of degree less than $n$.

For $f \in A$, we denote the degree of $f$ by $\operatorname{deg} f$, the number of polynomials $g$ coprime to $f$ with $\operatorname{deg} g<\operatorname{deg} f$ by $\phi(f)$, the number of divisors of $f$ by $d(f)$ and we set

$$
\sigma_{k}(f)=\sum_{\substack{d \in M \\ d \mid f}}|d|^{k}
$$

Finally, we set $|f|=q^{\operatorname{deg} f}$ if $f \neq 0$ and $|f|=0$ if $f=0$. It is well known (see [1]) that every $f \in A, f \neq 0$ can be written uniquely in the form

$$
f=\alpha \mathbf{p}_{1}^{e_{1}} \cdots \mathbf{p}_{s}^{e_{s}}
$$

where $\alpha$ is a nonzero element in $\mathbb{F}_{q}, \mathbf{p}_{1}, \ldots, \mathbf{p}_{s}$ are irreducible monic polynomials, $\mathbf{p}_{i} \neq \mathbf{p}_{j}$ for $i \neq j$, and $e_{1}, \ldots, e_{s}$ are nonnegative integers.

The following 2 propositions will be needed later. Their proofs are omitted here and can be found in [1]

Proposition 2. Let $f \in A$. If the prime decomposition of $f$ is given by

$$
\begin{aligned}
& f=\alpha \mathbf{p}_{1}^{e_{1}} \cdots \mathbf{p}_{s}^{e_{s}}, \text { then } \\
& \phi(f)=|f| \prod_{i=1}^{s}\left(1-\frac{1}{\left|\mathbf{p}_{i}\right|}\right) . \\
& d(f)=\left(e_{1}+1\right)\left(e_{2}+1\right) \cdots\left(e_{s}+1\right) . \\
& \sigma(f)=\frac{\left|\mathbf{p}_{1}\right|^{e_{1}+1}-1}{\left|\mathbf{p}_{1}\right|-1} \frac{\left|\mathbf{p}_{2}\right|^{e_{2}+1}-1}{\left|\mathbf{p}_{2}\right|-1} \cdots \frac{\left|\mathbf{p}_{s}\right|^{e_{s}+1}-1}{\left|\mathbf{p}_{s}\right|-1} .
\end{aligned}
$$

\section{Proposition 3.}

$$
\begin{aligned}
& \sum_{f \in M_{n}} d(f)=(n+1) q^{n} . \\
& \sum_{f \in M_{n}} \sigma(f)=q^{2 n} \cdot \frac{1-q^{-n-1}}{1-q^{-1}} .
\end{aligned}
$$

\section{Some Results about Sum of Reciprocals of Polynomials over $\mathbb{F}_{q}[t]$}

In this section, we give a closed formula for some sums of reciprocals of polynomials over $\mathbb{F}_{q}[t]$. These results will be used in the subsequent sections to help prove some polynomial identities in $\mathbb{F}_{q}[t]$.
We start with the following definitions. Letting $n \in \mathbb{N}$, we define $d_{n}$ and $l_{n}$ as

$$
\begin{aligned}
& d_{n}=\prod_{i=0}^{n-1}\left(t^{q^{n}}-t^{q^{i}}\right), \\
& l_{n}=\prod_{i=1}^{n}\left(t-t^{q^{i}}\right)
\end{aligned}
$$

Further, we set $d_{0}=l_{0}=1$, and, for an integer $k$, we define

$$
\begin{gathered}
S_{n}(k)=\sum_{a \in M_{n}} \frac{1}{a^{k}}, \\
S_{<n}(k)=\sum_{a \in M_{<n}} \frac{1}{a^{k}}
\end{gathered}
$$

The next 2 theorems are due to Carlitz [3]. The first one provides an explicit formula for $S_{n}(k)$ and $S_{<n}(k)$ if $k= \pm\left(q^{i}-\right.$ $1)$. Details of this theorem can be also found in [4].

Theorem 4 (Carlitz [3]). For each $n \in \mathbb{N}$, we have

$$
\begin{aligned}
S_{n}\left(q^{i}-1\right) & =\frac{l_{n+i-1}}{l_{i-1} l_{n}^{q^{i}}}, \\
S_{<n}\left(q^{i}-1\right) & =\frac{l_{n+i-1}}{l_{i} l_{n-1}^{q^{i}}}, \\
\text { for } i \geq n, & \geq n \\
S_{n}\left(-\left(q^{i}-1\right)\right) & =\frac{d_{i}}{l_{n} d_{i-n}^{q^{n}}}, \\
S_{<n}\left(-\left(q^{i}-1\right)\right) & =\frac{d_{i-1}^{q}}{l_{n-1} d_{i-n}^{q^{n}}}
\end{aligned}
$$

The next theorem is initially due to Carlitz [3], but an alternative proof was given by Hicks et al. [5] in a most recent paper published in 2012 .

Theorem 5 (Carlitz [3]). For $k \in \mathbb{N}, 1 \leq k \leq q$, we have

$$
\sum_{f \in M_{n}} \frac{1}{f^{k}}=\frac{1}{\left[\prod_{i=1}^{n}\left(t-t^{q^{i}}\right)\right]^{k}}=\frac{1}{l_{n}^{k}} .
$$

More results about sum of reciprocals of polynomials can be also found in [6]. We state here some of them. Let $R_{n}$ be the subset of all monic reducible polynomials in $A$ of degree less than $n$ and we denote by $h_{n}$ the product of all monic irreducible polynomials of degree $n$ in $A$, i.e.,

$$
h_{n}=\prod_{\mathbf{p} \in P_{n}} \mathbf{p} .
$$

S. Nelson [6] in 2016 has proved the following theorem. 
Theorem 6 (Nelson [6]). With $P_{n}, R_{n}$, and $h_{n}$ defined as above, we have

$$
\begin{aligned}
\sum_{\mathbf{p} \in P_{2}} \frac{1}{\mathbf{p}} & =-\frac{\left(t^{q}-t\right)^{q-3}}{h_{2}(t)} . \\
\sum_{\mathbf{p} \in P_{3}} \frac{1}{\mathbf{p}} & =\frac{\left(h_{2}(t)\right)^{3}\left(t^{q^{2}}-t\right)^{q-4}}{h_{3}(t)} . \\
\sum_{r \in R_{2}} \frac{1}{r} & =\frac{1}{\left(t^{q}-t\right)^{2}} . \\
\sum_{r \in R_{3}} \frac{1}{r} & =-\frac{1}{\left(t^{q^{2}}-t\right)\left(t^{q}-t\right)^{2}} .
\end{aligned}
$$

We conclude this section with our main theorem that states the following.

Theorem 7 (main theorem). Let $m \in M_{n}, 0 \neq a \in \mathbb{F}_{q}[t]$, and $b \in M_{<n}$. Assume $a=m s_{1}+r_{1}$ and $b^{u}=m s_{2}+r_{2}$, where $r_{i} \in M_{<n}, i=1,2$. Define

$$
\begin{aligned}
& v(f)=\sum_{b \in M_{<n}} \sum_{\substack{d \in M \\
d \mid f}}\left[\frac{|d| b^{u}+a}{m^{k}}\right], \\
& v_{1}(f)=\sum_{b \in M_{<n}} \sum_{\substack{d \in M \\
d \mid f}} \frac{|d| b^{u}+a}{m^{k}} .
\end{aligned}
$$

If $u=(|m|-1)|m|^{k-1}$, then

$$
\begin{aligned}
& \sum_{f \in M_{n}} v(f)=\frac{d_{n-1}^{|m|^{k-1}}}{m^{k} l_{n-1}^{|m|^{k-1}} d_{0}^{|m|^{k}}} q^{2 n} \cdot \frac{1-q^{-n-1}}{1-q^{-1}}-(n+1) \\
& \cdot q^{n}\left[\frac{1-|m|}{(1-q) m^{k}}\left(a-r_{1}\right)\right. \\
& \left.-\frac{1}{m^{k}} \sum_{b \in M_{<n}} \sum_{\substack{r_{2} \in M_{<n} \\
b^{|m|-1}=m s_{2}+r_{2}}} r_{2}\right],
\end{aligned}
$$

and

$$
\begin{aligned}
\sum_{f \in M_{n}} v_{1}(f)= & \frac{a}{m^{k}} \frac{q^{n}\left(1-q^{n}\right)\left(1-q^{n+1}\right)}{(1-q)^{2}} \\
& -(n+1) q^{n} \frac{d_{n-1}^{q}}{m^{k} l_{n-1}} .
\end{aligned}
$$

Theorem 7 will be used in Section 4 to establish some polynomials identities in $\mathbb{F}_{q}$. These identities are obtained by evaluating certain sums taken over the subset of all monic polynomials and monic irreducible polynomials.

\section{Polynomial Analogue of Some Liouville's Identities}

Let $k \in A$ and $m \in M$. We define the function $d_{k, m}(g)$ as the number of divisors $d$ of $g$ congruent to $k$ modulo $m$, i.e.,

$$
d_{k, m}(g):=\sum_{\substack{d \in M \\ d \mid g \\ d \equiv k(\bmod m)}} 1, \quad g \in M,
$$

The following theorem is the polynomial analogue of [2, theorem 3.8]. It describes some of the properties of $d_{k, m}$ for polynomials. The proof follows the same steps as the one for integers proved by Liouville in [2].

Lemma 8. For $m, g \in M$, we have

$$
\begin{aligned}
d_{0, m}(g) & =d\left(\frac{g}{m}\right), \\
\sum_{\substack{k \in M \\
\operatorname{deg} k<\operatorname{deg} m}} d_{k, m}(g) & =d(g) .
\end{aligned}
$$

Proof. The proof is essentially the same as the integer case. We have

$$
d_{0, m}(g)=\sum_{\substack{d \in M \\ d \mid g \\ d=0(\bmod m)}} 1=\sum_{\substack{e \in M \\ e m \mid g}} 1=\sum_{\substack{e \in M \\ e \mid g / m}} 1=d\left(\frac{g}{m}\right) .
$$

For $m=1$, we have

$$
d_{0,1}(g)=d(g)
$$

For the second equality, we have

$$
\begin{aligned}
\sum_{\substack{k \in M \\
\operatorname{deg} k<\operatorname{deg} m}} d_{k, m}(g) & =\sum_{\substack{k \in M \\
\operatorname{deg} k<\operatorname{deg} m}} \sum_{\substack{d \in M \\
d \mid g \\
d \equiv k(\bmod m)}} 1=\sum_{\substack{d \in M \\
d \mid g}} 1 \\
& =d(g) .
\end{aligned}
$$

Let $m, a \in \mathbb{F}_{q}[t]$. The division algorithm theorem states that that there exists a unique set of polynomials $s, r \in \mathbb{F}_{q}[t]$ satisfying $a=m s+r$, with $r=0$ or $\operatorname{deg} r<\operatorname{deg} m$. We define

$$
\left[\frac{a}{m}\right]=s \text {. }
$$

Remark 9. Since for $a, b \in \mathbb{F}_{q}[t], \operatorname{deg}(a+b)$ is the maximum between $\operatorname{deg} a$ and $\operatorname{deg} b$, for $m \in M$, we have

$$
\left[\frac{a+b}{m}\right]=\left[\frac{a}{m}\right]+\left[\frac{b}{m}\right] .
$$

Lemma 10 (see [2]). Let $0 \neq a, b \in \mathbb{F}_{q}[t]$ and $f, m \in M$. Then

$$
\sum_{\substack{b \in M \\ \operatorname{deg} b \leq \operatorname{deg} m}} \sum_{\substack{d \in M \\ d \mid f}} \frac{|d| a}{m}=\frac{(1-|m|)}{(1-q) m} a \sigma(f) .
$$


Proof. We have

$$
\begin{aligned}
\sum_{\substack{k \in M \\
\operatorname{deg} b \leq \operatorname{deg} m}} \sum_{\substack{d \in M \\
d \mid f}} \frac{|d| a}{m} & =\frac{a}{m} \sum_{\substack{b \in M \\
\operatorname{deg} b \leq \operatorname{deg} m}} \sum_{\substack{d \in M \\
d \mid f}}|d| \\
& =\frac{a}{m} \sum_{\substack{b \in M \\
\operatorname{deg} b \leq \operatorname{deg} m}} \sigma(f) \\
& =\frac{a}{m} \sigma(f) \sum_{\substack{\operatorname{deg} b \leq \operatorname{deg} m \\
\operatorname{deg} m}} 1 \\
& =\frac{a}{m} \sigma(f) \sum_{i=0}^{i} q^{i} \\
& =\frac{(1-|m|)}{(1-q) m} a \sigma(f) .
\end{aligned}
$$

The next theorem is the polynomial analogue of [2, theorem 3.9].

Theorem 11. Let $0 \neq a, b \in \mathbb{F}_{q}[t]$ and $m \in M$. If $\operatorname{gcd}(a, m)=1$ and $a a_{1} \equiv 1 \bmod m$, then

$$
\begin{aligned}
\sum_{\substack{d \in M \\
d \mid f}}\left[\frac{|d| a+b}{m}\right]= & \frac{a}{m} \sigma(f)+\frac{b}{m} d(f) \\
& -\frac{1}{m} \sum_{\substack{r \in M \\
\operatorname{deg} r<\operatorname{deg} m}} r d_{a_{1}(r-b), m} .
\end{aligned}
$$

Proof. For each $d \in M$, there exist a unique set of polynomials $s, r \in M$ such that $|d| a+b=m s+r, \operatorname{deg} r<\operatorname{deg} m$. Therefore, we have

$$
\begin{aligned}
\sum_{\substack{d \in M \\
d \mid f}}\left[\frac{|d| a+b}{m}\right]= & \sum_{s \in M} \sum_{\substack{d \in M \\
d \mid f \\
[(|d| a+b) / m]=s}} s \\
= & \sum_{\substack{r \in M \\
\operatorname{deg} r<\operatorname{deg} m}} \sum_{\substack{d \in M \\
d|f\\
| d \mid a+b=m s+r}} \frac{|d| a+b-r}{m} \\
= & \sum_{\substack{d \in M \\
d \mid f}} \frac{|d| a+b}{m} \sum_{\substack{r \in M \\
\operatorname{deg} r<\operatorname{deg} m}} \sum_{\substack{d \in M \\
d|f\\
| d \mid a+b=m s+r}} \frac{r}{m} \\
= & \frac{a}{m} \sum_{\substack{d \in M \\
d \mid f}}^{|d| d \mid+\frac{k}{m} \sum_{d \in M}} 1
\end{aligned}
$$

$$
\begin{gathered}
-\frac{1}{m} \sum_{\substack{r \in M \\
\operatorname{deg} r<\operatorname{deg} m}} r \sum_{\substack{d \in M \\
d|f\\
| d \mid a \equiv r-b \bmod m}} 1 \\
=\frac{a}{m} \sum_{d \in M}|d|+\frac{b}{m} \sum_{\substack{d \in M \\
d \mid f}} 1 \\
-\frac{1}{m} \sum_{\substack{r \in M \\
\operatorname{deg} r<\operatorname{deg} m}} r \sum_{\substack{d \in M \\
d \mid f \\
d \equiv h_{1}(r-b) \bmod m}} 1 \\
=\frac{a}{m} \sigma(f)+\frac{b}{m} d(f) \\
-\frac{1}{m} \sum_{\substack{r \in M \\
\operatorname{deg} r<\operatorname{deg} m}} r d_{a_{1}(r-b), m}(f) .
\end{gathered}
$$

Our last polynomial analogue result in this section deals with the function $F_{d}(a)$ defined for $f, d \in M$ as

$$
F_{d}(f)= \begin{cases}1, & \text { if } d \mid f \\ 0, & \text { if } d+f\end{cases}
$$

Lemma 12. For any $d_{1} \in M_{1}$,

$$
\sum_{f \in M_{n+1}} F_{d_{1}}(f)=\sum_{b \in M_{<n}} 1
$$

Proof. We have

$$
\begin{aligned}
\sum_{f \in M_{n+1}} F_{d_{1}}(f)= & \sum_{\substack{f \in M_{n+1} \\
d_{1} \mid f}} 1+\sum_{\substack{f \in M_{n+1} \\
d_{1} \nmid f}} 0 \\
= & \sum_{\substack{f \in M_{1} \\
d_{1} \mid f}} 1+\sum_{\substack{f \in M_{2} \\
d_{1} \mid f}} 1+\cdots+\sum_{\substack{f \in M_{n+1} \\
d_{1} \mid f}} 1+\sum_{\substack{f \in M_{2} \\
f=d_{1} \times e}} 1+\sum_{\substack{e \in M_{2} \\
f=d_{1} \times e}} 1+\cdots \\
= & +\sum_{\substack{f \in M_{n+1} \\
e \in M_{n} \\
f=d_{1} \times e}} 1=1+q+q^{2}+\cdots+q^{n} \\
= & \sum_{\substack{b \in M_{<n} \\
\sum_{<n}}} 1 .
\end{aligned}
$$

\section{Proof of the Main Theorem}

In this section, we prove the main Theorem 7 stated in Section 2. Some other sums are also evaluated. We need the following lemma. 
Lemma 13 (see [4]). Let $0 \neq a, b \in \mathbb{F}_{q}[t]$ and $f, m \in M$. Then

$$
\begin{gathered}
\sum_{\substack{b \in M \\
\operatorname{deg} b<\operatorname{deg} m}} \sum_{\substack{d \in M \\
d \mid f}} \frac{|d| b^{(|m|-1)|m|^{k-1}}}{m^{k}} \\
=\frac{d_{n-1}^{|m|^{k-1}}}{l_{n-1}^{|m|^{k-1}} d_{0}^{|m|^{k}}}\left(\frac{\sigma(f)}{m^{k}}\right) .
\end{gathered}
$$

Proof. We have

$$
\begin{aligned}
& \sum_{\substack{b \in M \\
\operatorname{deg} b<\operatorname{deg} m}} \sum_{\substack{d \in M \\
d \mid f}} \frac{|d| b^{(|m|-1)|m|^{k-1}}}{m^{k}} \\
& =\frac{1}{m^{k}} \sum_{\substack{b \in M \\
\operatorname{deg} b<\operatorname{deg} m}} b^{(|m|-1)|m|^{k-1}} \sum_{\substack{d \in M \\
d \mid f}}|d| \\
& =\frac{\sigma(f)}{m^{k}} \sum_{\substack{b \in M \\
\operatorname{deg} b<\operatorname{deg} m}} b^{(|m|-1)|m|^{k-1}} \\
& =\frac{\sigma(f)}{m^{k}}\left(\sum_{\substack{b \in M \\
\operatorname{deg} b<\operatorname{deg} m}} b^{(|m|-1)}\right)^{|m|^{k-1}} .
\end{aligned}
$$

Therefore, by Theorem 4 the proof is complete.

\subsection{Proof of the Main Theorem}

Proof. We have $|d| b^{u}+a=\left(|d| s_{2}+s_{1}\right) m^{k}+\left(|d| r_{2}+r_{1}\right)$ and

$$
\left[\frac{|d| b^{u}+a}{m^{k}}\right]=\left[\frac{|d| b^{u}}{m^{k}}\right]+\left[\frac{a}{m^{k}}\right]
$$

Therefore, we have

$$
\begin{aligned}
& v(f)=\sum_{b \in M_{<n}} \sum_{\substack{d \in M \\
d \mid f}}\left[\frac{|d| b^{u}+a}{m^{k}}\right] \\
& =\sum_{b \in M_{<n}} \sum_{\substack{d \in M \\
d \mid f}}\left[\frac{|d| b^{u}}{m^{k}}\right]+\sum_{b \in M_{<n}} \sum_{\substack{d \in M \\
d \mid f}}\left[\frac{a}{m^{k}}\right] \\
& =\sum_{b \in M_{<n}} \sum_{s_{2} \in M} \sum_{\begin{array}{c}
d \in M \\
d \mid f \\
{\left[|d| b^{u} / m^{k}\right]=|d| s_{2}}
\end{array}}|d| s_{2} \\
& +\sum_{b \in M_{<n}} \sum_{s_{1} \in M} \sum_{\substack{d \in M \\
d \mid f \\
\left[a / m^{k}\right]=s_{1}}} s_{1}
\end{aligned}
$$

Rewriting the summation in terms of $r_{1}$ and $r_{2}$ yields

$$
\begin{aligned}
v(f)= & \sum_{b \in M_{<n}} \sum_{\substack{d \in M \\
d \mid f \\
a=m s_{1}+r_{1}}} \frac{a-r_{1}}{m^{k}} \\
& +\sum_{b \in M_{<n}} \sum_{r_{2} \in M_{<n}} \sum_{\begin{array}{c}
d \in M \\
d \mid f \\
b^{u}=m s_{2}+r_{2}
\end{array}} \frac{|d| b^{u}-r_{2}}{m^{k}} .
\end{aligned}
$$

Hence Lemmas 10 and 13 imply

$$
\begin{aligned}
& v(f)=\frac{1-|m|}{(1-q) m^{k}}\left(a-r_{1}\right) d(f) \\
& +\frac{d_{n-1}^{|m|^{k-1}}}{l_{n-1}^{|m|^{k-1}} d_{0}^{|m|^{k}}}\left(\frac{\sigma(f)}{m^{k}}\right)-\frac{d(f)}{m^{k}} \\
& \cdot \sum_{b \in M_{<n}} \sum_{\substack{r_{2} \in M_{<n} \\
b^{|m|^{-1}}=m s_{2}+r_{2}}} r_{2}=\frac{d_{n-1}^{|m|^{k-1}}}{l_{n-1}^{|m|^{k-1}} d_{0}^{|m|^{k}}}\left(\frac{\sigma(f)}{m^{k}}\right) \\
& +d(f)\left[\frac{1-|m|}{(1-q) m^{k}}\left(a-r_{1}\right)\right. \\
& \left.-\frac{1}{m^{k}} \sum_{b \in M_{<n}} \sum_{\substack{r_{2} \in M_{<n} \\
b^{m \mid-1}=m s_{2}+r_{2}}} r_{2}\right] .
\end{aligned}
$$

Therefore,

$$
\sum_{f \in M_{n}} v(f)=\frac{d_{n-1}^{|m|^{k-1}}}{m^{k} l_{n-1}^{|m|^{-1}} d_{0}^{|m|^{k}}} \sum_{f \in M_{n}} \sigma(f)
$$

$$
\begin{aligned}
& -\left[\frac{1-|m|}{(1-q) m^{k}}\left(a-r_{1}\right)-\frac{1}{m^{k}} \sum_{b \in M_{<n}} \sum_{\substack{r_{2} \in M_{<n} \\
b^{u}=m^{k} s_{2}+r_{2}}} r_{2}\right. \\
& \cdot \sum_{f \in M_{n}} d(f)=\frac{d_{n-1}^{|m|^{k-1}}}{m^{k} l_{n-1}^{\left.|m|\right|^{k-1}} d_{0}^{|m|^{\mid}} q^{2 n} \cdot \frac{1-q^{-n-1}}{1-q^{-1}}-(n} \\
& +1) q^{n}\left[\begin{array}{c}
\frac{1-|m|}{(1-q) m^{k}}\left(a-r_{1}\right) \\
-\frac{1}{m^{k}} \sum_{b \in M_{<n}} \sum_{\substack{r_{2} \in M_{<n} \\
b^{u}=m^{k} s_{2}+r_{2}}} r_{2}
\end{array}\right] .
\end{aligned}
$$


The proof of (18) is similar and is a direct consequence of Lemma 13.

Corollary 14. If $m=\mathbf{p} \in \mathbf{P}_{\mathbf{n}}$, then

$$
\begin{aligned}
& \sum_{f \in M_{n}} v(f) \\
& =\frac{d_{n-1}^{|\mathbf{p}|^{\mathbf{k}-1}}}{l_{n-1}^{|\mathbf{p}|^{\mathbf{k}-1}} d_{0}^{\mid \mathbf{p}^{\mathbf{k}}} \mathbf{p}^{\mathbf{k}}} q^{2 n} \cdot \frac{1-q^{-n-1}}{1-q^{-1}} \\
& -(n+1) q^{n}\left[\frac{1-|\mathbf{p}|}{(1-q) \mathbf{p}^{\mathbf{k}}}\left(a-r_{1}\right)-\frac{1}{\mathbf{p}^{\mathbf{k}}} \frac{1-|\mathbf{p}|}{(1-q)}\right] \text {. }
\end{aligned}
$$

Proof. If $\in P_{n}$, then, for each $b \in M_{<n}, b^{u}=\mathbf{p}^{\mathbf{k}} s_{2}+r_{2}$, we have

$$
b^{u}=b^{(|\mathbf{p}|-1)|\mathbf{p}|^{\mathbf{k}-1}} \equiv r_{2} \bmod \mathbf{p}^{\mathbf{k}},
$$

which forces $r_{2}=1$ by Euler theorem for polynomial; see [1]. Therefore,

$$
\sum_{b \in M_{<n}} \sum_{\substack{r_{2} \in M_{<n} \\ b^{|\mathbf{p}|-1}=p s_{2}+r_{2}}} r_{2}=\frac{1-|\mathbf{p}|}{(1-q)},
$$

and the corollary follows from the main theorem.

\subsection{Evaluation of Some Sums in $\mathbb{F}_{q}[t]$}

Theorem 15. Let $n \in \mathbb{N}$ and for $k \in \mathbb{N}$.

$$
\begin{aligned}
& \text { If } 1 \leq k \leq q \text {, then } \\
& \sum_{m \in M_{n}} \sum_{f \in M_{n}} v_{1}(f) \\
& \quad=a\left[\frac{q^{n}\left(1-q^{n}\right)\left(1-q^{n+1}\right)}{(1-q)^{2}}-(n+1) q^{n} \frac{d_{n-1}^{q}}{l_{n-1}}\right] \frac{1}{l_{n}^{k}} .
\end{aligned}
$$

Proof. Using the main theorem, we obtain

$$
\begin{aligned}
\sum_{m \in M_{n}} \sum_{f \in M_{n}} v_{1}(f)= & \sum_{m \in M_{n}} \frac{a}{m^{k}} \frac{q^{n}\left(1-q^{n}\right)\left(1-q^{n+1}\right)}{(1-q)^{2}} \\
& -(n+1) q^{n} \frac{d_{n-1}^{q}}{m^{k} l_{n-1}} .
\end{aligned}
$$

Therefore, if $1 \leq k \leq q$, Theorem 5 implies

$$
\begin{aligned}
& \sum_{m \in M_{n}}\left[\frac{a}{m^{k}} \frac{q^{n}\left(1-q^{n}\right)\left(1-q^{n+1}\right)}{(1-q)^{2}}\right. \\
& \left.-(n+1) q^{n} \frac{d_{n-1}^{q}}{m^{k} l_{n-1}}\right]=a\left[\frac{q^{n}\left(1-q^{n}\right)\left(1-q^{n+1}\right)}{(1-q)^{2}}\right. \\
& \left.-(n+1) q^{n} \frac{d_{n-1}^{q}}{l_{n-1}}\right] \frac{1}{l_{n}^{k}} .
\end{aligned}
$$

which complete the proof of (43).
Theorem 16. For $k=1$, we have

$$
\begin{aligned}
\sum_{m \in P_{2}} & \sum_{f \in M_{n}} v_{1}(f) \\
= & a \frac{\left(t^{q}-t\right)^{q-3}}{h_{2}(t)} \frac{q^{n}\left(1-q^{n}\right)\left(1-q^{n+1}\right)}{(1-q)^{2}} \\
& +(n+1) q^{n}\left[\frac{d_{n-1}^{q}}{l_{n-1}} \frac{\left(t^{q}-t\right)^{q-3}}{h_{2}(t)}\right] . \\
\sum_{m \in P_{3}} & \sum_{f \in M_{n}} v_{1}(f) \\
= & a \frac{\left(h_{2}(t)\right)^{3}\left(t^{q^{2}}-t\right)^{q-4}}{h_{3}(t)} \frac{q^{n}\left(1-q^{n}\right)\left(1-q^{n+1}\right)}{(1-q)^{2}} \\
& -(n+1) q^{n}\left[\frac{d_{n-1}^{q}}{l_{n-1}} \frac{\left(h_{2}(t)\right)^{3}\left(t^{q^{2}}-t\right)^{q-4}}{h_{3}(t)}\right] .
\end{aligned}
$$

Proof. By the main theorem, we have

$$
\begin{aligned}
\sum_{m \in P_{2}} \sum_{f \in M_{n}} v_{1}(f)= & \sum_{m \in P_{2}} \frac{a}{m^{k}} \frac{q^{n}\left(1-q^{n}\right)\left(1-q^{n+1}\right)}{(1-q)^{2}} \\
& -(n+1) q^{n} \frac{d_{n-1}^{q}}{m^{k} l_{n-1}} .
\end{aligned}
$$

Therefore, if $k=1$, then Theorem 6 implies

$$
\begin{aligned}
\sum_{m \in P_{2}} & {\left[\frac{a}{m^{k}} \frac{q^{n}\left(1-q^{n}\right)\left(1-q^{n+1}\right)}{(1-q)^{2}}-(n+1) q^{n} \frac{d_{n-1}^{q}}{m^{k} l_{n-1}}\right] } \\
= & {\left[a \frac{q^{n}\left(1-q^{n}\right)\left(1-q^{n+1}\right)}{(1-q)^{2}}+(n+1) q^{n} \frac{d_{n-1}^{q}}{l_{n-1}}\right] } \\
& \cdot \frac{\left(t^{q}-t\right)^{q-3}}{h_{2}(t)} .
\end{aligned}
$$

which completes the proof of (46). The proof of (47) is similar and follows directly from the main theorem and Theorem 6 of Nelson.

Theorem 17. For $k=1$, we have

$$
\begin{aligned}
& \sum_{m \in R_{2}} \sum_{f \in M_{n}} v_{1}(f) \\
& =\left[a \frac{q^{n}\left(1-q^{n}\right)\left(1-q^{n+1}\right)}{(1-q)^{2}}-(n+1) q^{n} \frac{d_{n-1}^{q}}{l_{n-1}}\right] \\
& \cdot \frac{1}{\left(t^{q}-t\right)^{2}} .
\end{aligned}
$$




$$
\begin{aligned}
\sum_{m \in R_{3}} & \sum_{f \in M_{n}} v_{1}(f) \\
= & {\left[(n+1) q^{n} \frac{d_{n-1}^{q}}{l_{n-1}}-a \frac{q^{n}\left(1-q^{n}\right)\left(1-q^{n+1}\right)}{(1-q)^{2}}\right] } \\
& \cdot \frac{1}{\left(\left(t^{q^{2}}-t\right)\left(t^{q}-t\right)^{2}\right.} .
\end{aligned}
$$

Proof. Using the main theorem, we obtain

$$
\begin{aligned}
\sum_{m \in R_{2}} \sum_{f \in M_{n}} v_{1}(f)= & \sum_{m \in R_{2}} \frac{a}{m^{k}} \frac{q^{n}\left(1-q^{n}\right)\left(1-q^{n+1}\right)}{(1-q)^{2}} \\
& -(n+1) q^{n} \frac{d_{n-1}^{q}}{m^{k} l_{n-1}} .
\end{aligned}
$$

Therefore, if $k=1$, then Theorem 6 implies

$$
\begin{aligned}
& \sum_{m \in M_{n}}\left[\frac{a}{m^{k}} \frac{q^{n}\left(1-q^{n}\right)\left(1-q^{n+1}\right)}{(1-q)^{2}}\right. \\
& \left.-(n+1) q^{n} \frac{d_{n-1}^{q}}{m^{k} l_{n-1}}\right]=a\left[\frac{q^{n}\left(1-q^{n}\right)\left(1-q^{n+1}\right)}{(1-q)^{2}}\right. \\
& \left.-(n+1) q^{n} \frac{d_{n-1}^{q}}{l_{n-1}}\right] \frac{1}{\left.t^{q}-t\right)^{2}} .
\end{aligned}
$$

which complete the proof of (50). The proof of (51) is similar and follows directly from the main theorem and Theorem 6 of Nelson.

Example 18. In this example, we let $m$ runs through the set of all linear polynomials. then

Let $n \in \mathbb{N}$ and $k \in \mathbb{N}$. If $1 \leq k \leq q$ and $u=(|m|-1)|m|^{k-1}$,

$$
\sum_{m \in M_{1}} \sum_{f \in M_{n}} \sum_{b \in M_{<n}} \sum_{\substack{d \in M \\ d \mid f}} \frac{|d| b^{u}+a}{m^{k}}=\frac{a q(q-1)}{\left(t-t^{q}\right)^{k}} .
$$

\section{Conclusion}

In this project, we prove the polynomial analogue of some Liouville theorems involving the arithmetic polynomial divisor functions. We then use the results obtained to establish several polynomials identities over the finite fields of $q$ elements. As an example, we show that some complex sums of polynomials in $\mathbb{F}_{q}[t]$ may be reduced to very simple algebraic expressions.

\section{Data Availability}

No data were used to support this study.

\section{Conflicts of Interest}

The author declares that there are no conflicts of interest.

\section{Acknowledgments}

An earlier version of this paper was presented in 12th Annual International Conference on Mathematics and Statistics: Teaching, Theory and Applications, which took place in July 2018 in Athens, Greece. The author would like to sincerely thank and encourage the three undergraduate students (Tasnim I. Kreishan, Nada M. Alsereidi, and Fatima A. Albreiki) for their involvement in the project during the summer and fall 2017. The author is also very grateful to the Research and Sponsored Office at the United Arab Emirates University for accepting to fund this project. This work was supported by the Summer Undergraduate Research Program [SURE PLUS 2017] at the United Arab Emirates University [Grant 31S267 (G00002400)].

\section{References}

[1] M. Rosen, Number Theory in Function Fields, vol. 2010 of Graduate Texts in Mathematics, Springer, 2002.

[2] K. S. Williams, Number Theory in the Spirit of Liouville, Cambridge University Press, NY, USA, 2011.

[3] L. Carlitz, "On certain functions connected with polynomials in a Galois field," Duke Mathematical Journal, vol. 1, no. 2, pp. 137-168, 1935.

[4] D. S. Thakur, "Power sums of polynomials over finite fields and applications: a survey," Finite Fields and Their Applications, vol. 32, pp. 171-191, 2015.

[5] K. Hicks, X.-d. Hou, and G. L. Mullen, "Sums of reciprocals of polynomials over finite fields," The American Mathematical Monthly, vol. 119, no. 4, pp. 313-317, 2012.

[6] S. Nelson, "Sums of reciprocals of irreducible polynomials over finite fields," Rose-Hulman Undergraduate Mathematics Journal, vol. 17, no. 2, article no. 2, 2016. 


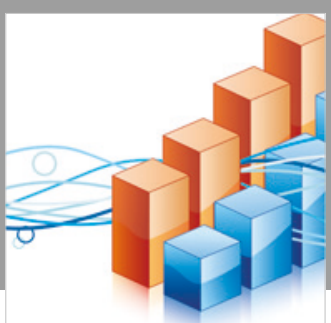

Advances in

Operations Research

\section{-n-m}
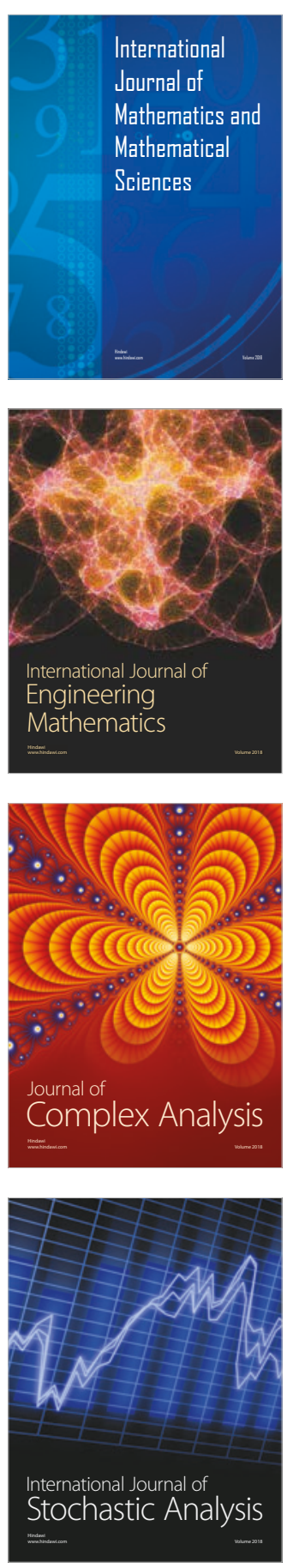
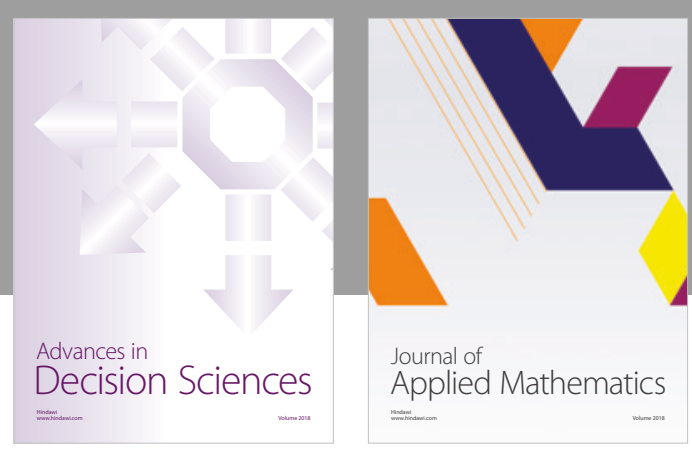

Journal of

Applied Mathematics
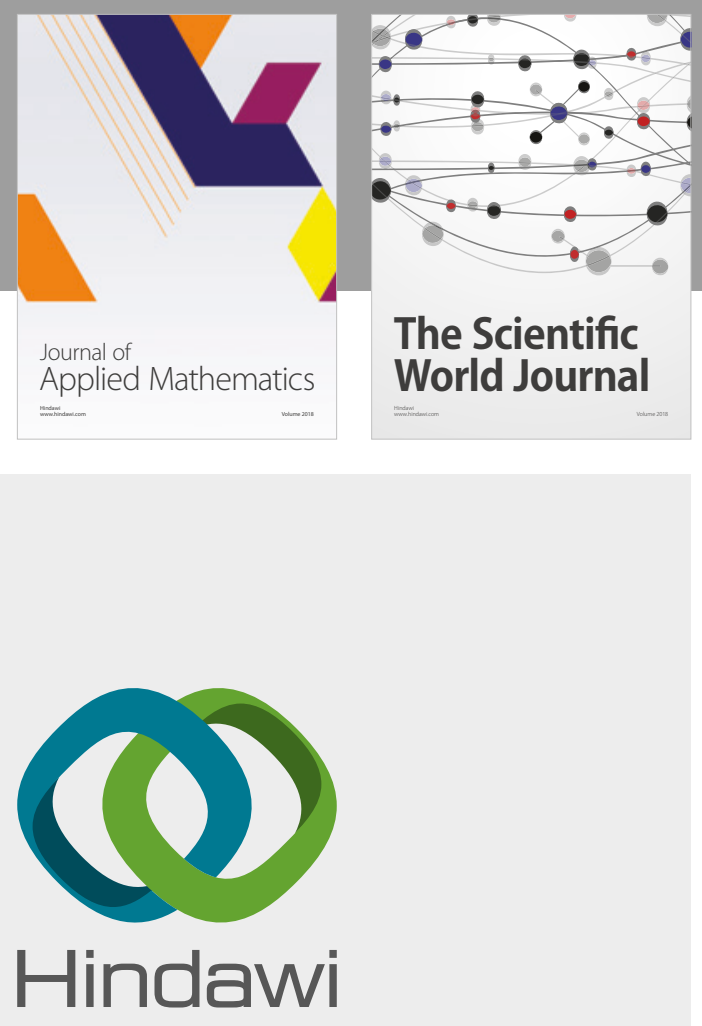

Submit your manuscripts at

www.hindawi.com

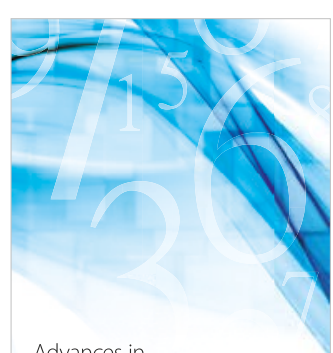

Advances in
Numerical Analysis
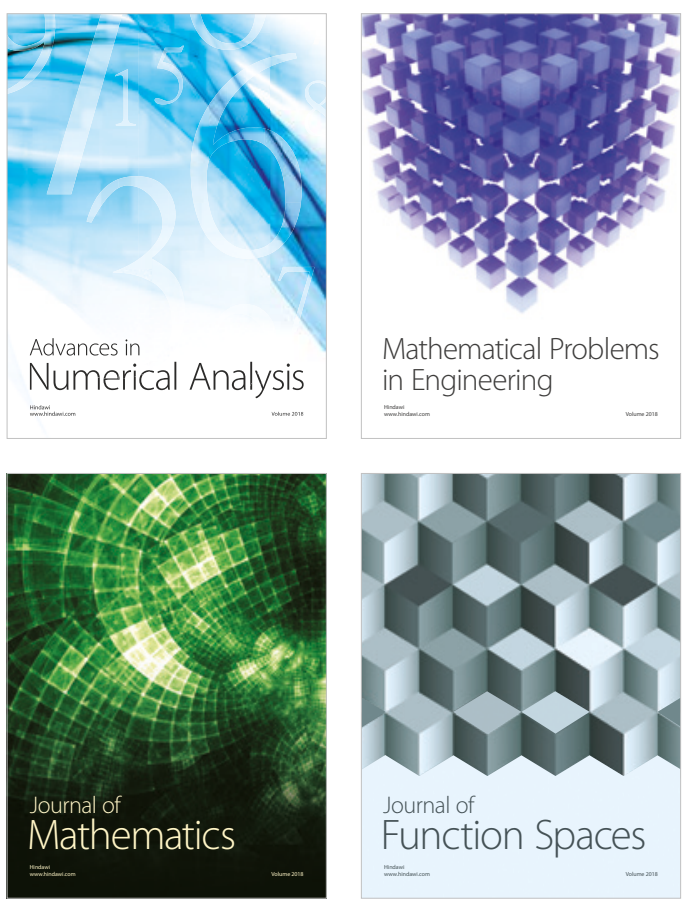

Mathematical Problems in Engineering

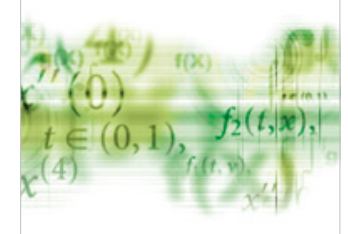

International Journal of

Differential Equations

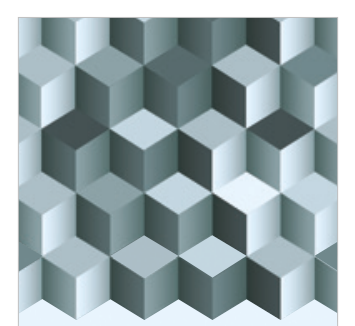

Journal of

Function Spaces
The Scientific

World Journal

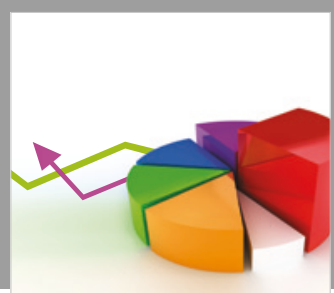

Journal of

Probability and Statistics
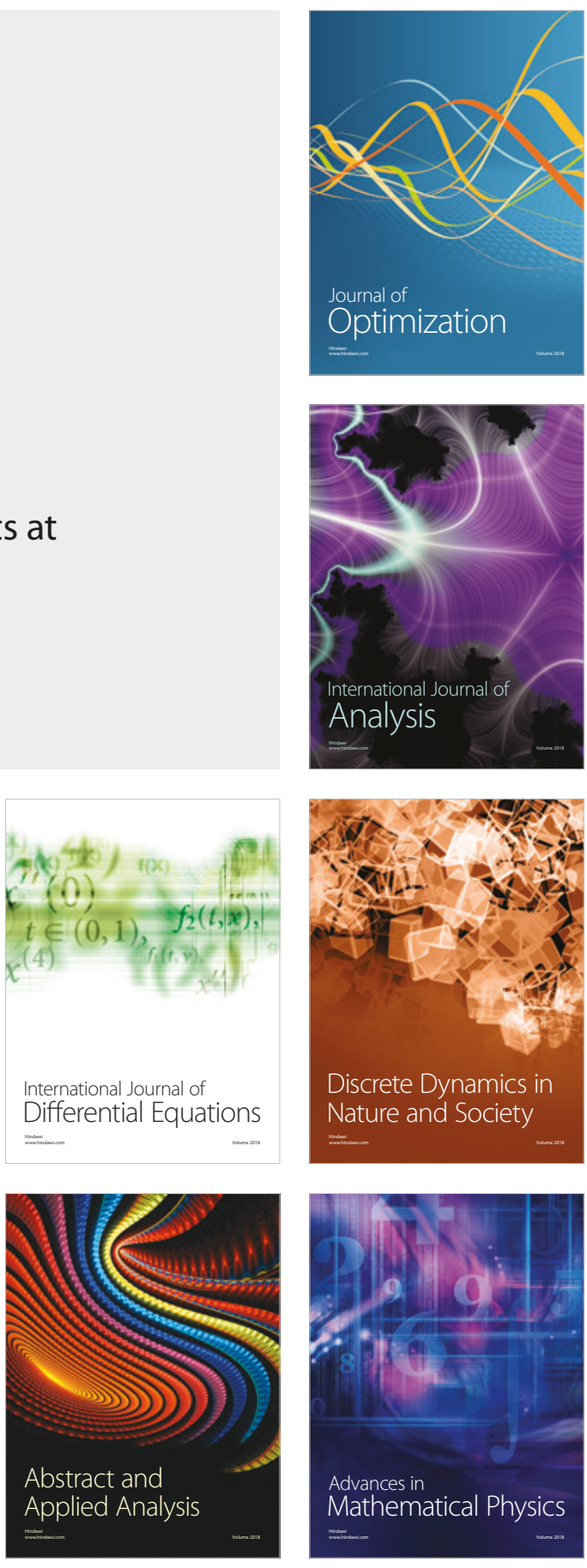